\title{
Evaluation of clinical outcomes of implants placed into the maxillary sinus with a perforated sinus membrane: a retrospective study
}

Gwang-Seok Kim, Jae-Wang Lee, Jong-Hyon Chong, Jeong Joon Han, Seunggon Jung, Min-Suk Kook, Hong-Ju Park, Sun-Youl Ryu and Hee-Kyun Oh ${ }^{*}$

\begin{abstract}
Background: The aim of this study was to evaluate the clinical outcomes of implants that were placed within the maxillary sinus that has a perforated sinus membrane by the lateral window approach.

Methods: We examined the medical records of the patients who had implants placed within the maxillary sinus that has a perforated sinus membrane by the lateral approach at the Department of Oral and Maxillofacial Surgery of Chonnam National University Dental Hospital from January 2009 to December 2015. There were 41 patients (male:female $=28: 13$ ). The mean age of patients was $57.2 \pm 7.2$ years at the time of operation (range, 20-76 years). The mean follow-up duration was 2.1 years (range, $0.5-5$ years) after implant placement. Regarding the method of sinus elevation, only the lateral approach was included in this study.

Results: Ninety-nine implants were placed in 41 patients whose sinus membranes were perforated during lateral approach. The perforated sinus membranes were repaired with a resorbable collagen membrane. Simultaneous implant placements with sinus bone grafting were performed in 37 patients, whereas delayed placements were done in four patients. The average residual bone height was $3.4 \pm 2.0 \mathrm{~mm}$ in cases of simultaneous implant placement and $0.6 \pm 0.9 \mathrm{~mm}$ in cases of delayed placement. Maxillary bone graft with implant placement, performed on the patients with a perforated maxillary sinus membrane did not fail, and the cumulative implant survival rate was $100 \%$.
\end{abstract}

Conclusions: In patients with perforations of the sinus mucosa, sinus elevation and implant placement are possible regardless of the location and size of membrane perforation. Repair using resorbable collagen membrane is a predictable and reliable technique.

Keywords: Perforation of sinus membrane, Resorbable collagen membrane, Survival rate of implant, Perforation repair, Complications after bone graft

\section{Background}

Maxillary sinus elevation, also known as maxillary sinus lift, is regarded as a standardized technique for the recovery of the masticatory function followed by placing implants in the atrophic maxillary posterior region. Elevation of the Schneiderian membrane in the maxillary sinus is a very delicate procedure; thus, perforation of

\footnotetext{
* Correspondence: hkoh@jnu.ac.kr

Department of Oral and Maxillofacial Surgery, School of Dentistry, Chonnam National University, 77, Yongbong-ro, Buk-gu, Gwangju, Republic of Korea
}

the mucosa during an operation occurs frequently (1055\%) [1-5]. Perforation of the membrane makes a direct communication to the maxillary sinus, and via this communication the graft material can be scattered into the sinus space; however, it also can be the cause of infection or sinusitis. Vlassis et al. [6] reported that sinus perforation occurs during the process of osteotomy when forming the window rather than the process of separating the mucosa from the bony wall. They also reported that smokers show a higher perforation rate 
than non-smokers and other factors, such as antral septa and narrow sinus, can lead to perforation of the maxillary sinus. Though anatomical factors can be overcome by the operator, predisposing causes towards the sinus perforation, such as smoking, is important for prevention. In addition, history of previous maxillary sinus operation can lead to a large perforation [4, 7]. Ardekian et al. [8] reported $85 \%$ perforation rate of sinus membranes with a residual ridge of $3 \mathrm{~mm}$, while $25 \%$ perforation rate for residual ridge of $6 \mathrm{~mm}$.

Small perforations $(<5 \mathrm{~mm})$ at the site of folded mucosa are reported to be healed by itself $[4,9]$. However, if the perforation is large $(\geq 5 \mathrm{~mm})$ and located on an unfavorable site such as the middle third of the lower marginal area of the lateral window, the perforation requires repair by a collagen membrane $[2,4,7,8,10,11]$, fibrin adhesive [12], block graft [6,9], or suturing using resorbable material [12], in order to prevent the loss of graft material. If the perforation cannot be blocked, the procedure should be discontinued.

If the perforated sinus membrane is treated properly, there is no adverse effect on the survival of the implant placed in a perforated maxillary sinus [7]. However, few studies have investigated the clinical outcomes and survival rate of these procedures. The aim of this study was to evaluate the clinical outcomes of implants, which were placed after sinus elevation of a perforated maxillary sinus membrane.

\section{Methods}

\section{Patients}

This retrospective study reviewed the medical records of cases of maxillary sinus perforations that occurred during a sinus lift procedure at the Department of Oral and Maxillofacial Surgery, Chonnam National University Dental Hospital from January 2009 to December 2015. All surgical procedures were done by one oral and maxillofacial surgeon. This study included 41 patients (male:female $=28: 13$; mean age, $57.2 \pm 7.2$ years; age range, 20-76 years). Mean follow-up period was 2.1 years (range, $0.5-5$ years) after implant placement. The study excluded patients who went to other dental clinics for prosthetic restoration and were absent from follow-up appointment without notice. Maxillary sinus elevation was performed using the lateral approach. Perforation of the mucosa was immediately documented in the medical records.

As the clinical setting of the repair for the perforated membrane, if the size of the perforation was more than $5 \mathrm{~mm}$ or the graft material was expected to be scattered via the perforation, the repair was performed. Implants were placed simultaneously with the sinus floor augmentation if the residual bone height was $>2 \mathrm{~mm}$, and initial stability of the implant was observed. Initial stability was confirmed clinically by the surgeon based on the mobility of the implant fixture. Otherwise, the implant was placed more than 4 months (maturation period of the graft material) after the sinus floor augmentation. There were no immediately loaded implants.

In this study, we evaluated the following parameters: (1) distribution of age and gender; (2) types of graft materials for maxillary sinus bone graft; (3) timing of implant placement by residual bone height; (4) barrier membrane used for the repair of perforated sinus; (5) types of implant surfaces; (6) complications after bone graft; (7) timing of loading after implant placement; and (8) survival rates.

\section{Surgical technique}

When the perforation of the maxillary sinus membrane was encountered during any case with a lateral approach, the bony window was widened with a Kerrison rongeur to expose the perforated sinus membrane after which the membrane was separated from sinus wall and elevated carefully (Figs. 1 and 2). After complete elevation of the perforated sinus membrane, the implant placement sites were prepared according to the protocol of the implant manufacturer while protecting the torn membrane. The autogenous bone, such as the maxillary tuberosity or ramus, was harvested and morcellized. The particulated bone was usually mixed with allograft or xenograft, and the fibrin sealant was injected onto the mixture of bone grafts.

The perforated membrane was repaired with the collagen membrane and fibrin sealant; bone graft materials were packed into the sinus under the collagen tape and implants were simultaneously placed into the prepared sites. Subsequently, the cover screws were connected. If primary stability could not be obtained, the implant placement was delayed approximately 4 months after sinus elevation. Sometimes, additional bone grafts were performed through the window of the lateral wall. The incised wound was sutured with 3-0 Mersilk (Ethicon Inc., Somerville, NJ, USA).

\section{Postoperative management}

All patients were administered antibiotics (Augmentin Ilsung pharmaceuticals Co., Seoul, Korea) thrice a day for 3 days after the surgery. In case of postoperative infection or maxillary sinusitis, antibiotics were prescribed for one to two additional weeks. The patients were given instructions to avoid elevating air pressure on the maxillary sinus; such as, "do not blow your nose" or "to sneeze with your mouth open," for 2 weeks.

After surgery, all patients underwent periapical and panoramic radiography. There was no immediate loading on the implants. Implant prostheses were usually restored after 6 months (mean $=6.5$ months; range: 5 to 12 months). 


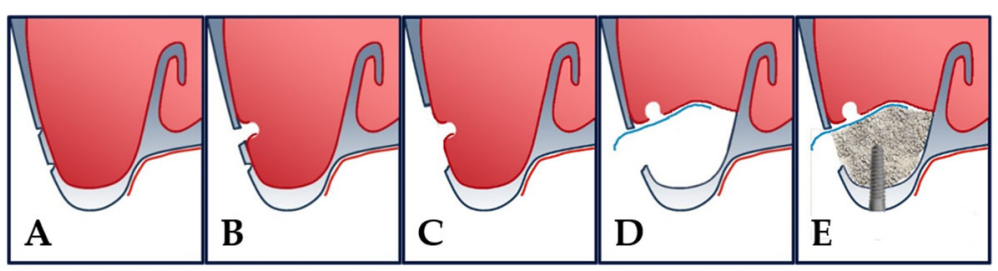

Fig. 1 Schematic drawing of sinus perforation repair using an absorbable membrane. a Procedure of making the lateral window. $\mathbf{b}$ Occurrence of perforation. $\mathbf{c}$ Widening of the window. $\mathbf{d}$ Repair of the perforated membrane with an absorbable collagen membrane. e Bone graft in the elevated sinus and simultaneous implant placement

\section{Results}

\section{Distribution of age and gender}

A total of 99 implants were placed in 41 patients $(28$ men and 13 women). The ages ranged from 20 to 76 years (mean age, $57.2 \pm 10.5$ years).

\section{Types of graft materials for maxillary sinus bone graft}

Mandibular ramus (19 patients) and maxillary tuberosity (13 patients) were used alone or in combination with other graft materials. Bio-Oss (Geistlich Pharma $\mathrm{AG}^{\circ}$, Wolhusen, Switzerland) was used as the xenograft in 16 patients. Ora-graft (DFDB - LifeNet Health', Virginia Beach, VA, USA) was used as the allograft in 9 patients, and Novosis (CGBio ${ }^{\circ}$ Seongnam, Korea) was used as the alloplast in 1 patient (Table 1).

\section{Timing of implant placement by residual bone height}

The preoperative dental CT images showed that the average residual bone height was $3.2 \pm 1.9 \mathrm{~mm}$ (range, 0-9.7 mm), $3.4 \pm 2.0 \mathrm{~mm}$ (range, $0-9.7 \mathrm{~mm}$ ) in cases of simultaneous implant placement, and $0.6 \pm 0.9 \mathrm{~mm}$ (range, $0-2.2 \mathrm{~mm}$ ) in delayed implant placement. Of the 99 implants placed, 7 delayed implants were performed in 4 patients with alveolar crest bone heights $<3 \mathrm{~mm}$. The remaining implants were placed simultaneously during the sinus elevation (Table 2).

\section{Barrier membranes used to repair the perforated sinus} Four types of resorbing collagen membranes were used for repair of the perforated sinus membrane; Rapiderm tape (Dalimtissen', Seoul, Korea) in 23 patients, Ossguide (Osstem, Seoul, Korea) in 12 patients, Collatape (Zimmer Biomet, Warsaw, Indiana, USA) in 3 patients, and Geistlich Bio-Gide (Geistlich Pharma AG $^{\circ}$, Wolhusen, Switzerland) in 3 patients.

\section{Types of implant surfaces}

In this study, 57 resorbable blasting media (RBM) implants (52 Osstem,' Osstem Co., Seoul, Korea, 5 Megagen, Megagen Co., Gyeongsan, Korea), 39 sand blasted with alumina and acid-etching (SA) implants (Osstem ', Osstem Co., Seoul, Korea), and 3 sand-blasted and acid etched (S\&E) implants (Luna, Shinhung Co., Seoul, Korea) were used. Regarding the timing of implant placement, 92 implants were placed simultaneously with sinus elevation in 37 patients and 7 implants were placed in 4 patients with delayed implantation 3 4 months after sinus augmentation (Table 3).

\section{Complications after bone graft}

The common postoperative complications of a maxillary sinus lift and implant placement includes edema and pain at the operation site and the time needed for relief from the complications. Among 41 patients, 14 patients (34.1\%) were prescribed additional antibiotics due to postoperative infection or maxillary sinusitis. Eight patients had mild sinusitis based on radiographic findings (Waters' view) and were prescribed metronidazole together with amoxicillin/clavulanic acid for an additional week. Infection symptoms (e.g., pain, swelling,

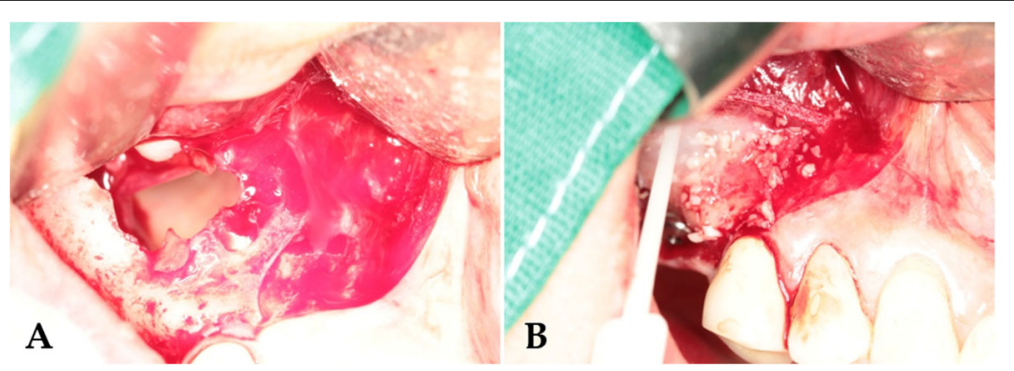

Fig. 2 Intraoperative photographs. a Confirmation of the perforation in the sinus membrane. $\mathbf{b}$ Repair using collagen tape and packing of the graft material with fibrin adhesive 
Table 1 Types of graft materials for the maxillary sinus graft

\begin{tabular}{ll}
\hline Graft materials & Number of patients \\
\hline llium only & 1 \\
llium + xenograft & 2 \\
Ramus only & 8 \\
Ramus + xenograft & 8 \\
Ramus + allograft & 1 \\
Ramus + tuberosity + allograft & 1 \\
Tuberosity only & 6 \\
Tuberosity + allograft & 4 \\
Tuberosity + xenograft & 2 \\
Xenograft only & 4 \\
Allograft only & 3 \\
Alloplast only & 1 \\
Total & 41 \\
\hline
\end{tabular}

localized fever on operative region) were observed in the other six patients without radiographic sign. In these cases, we prescribed amoxicillin/clavulanic acid for an additional week. Nasal congestion or rhinorrhea was present in 10 patients who were prescribed pseudoephedrine (Sudafed, Johnson \& Johnson Co., USA) for a week.

\section{Timing of loading after implant placement}

There was no immediate loading on the implants. Implant loading was performed at an average 6.5 months after implant placement (range, 5-12 months).

\section{Survival rates}

Patients with implant placement in the perforated sinus membrane showed no failure of implant. All implants included in the study showed $100 \%$ survival.

\section{Discussion}

Hernandez-Alfaro et al. [13] classified perforations into three types depending on the size of perforation. Fugazzotto and Vlassis [10] classified them all into four types, including three types based on the location with two subtypes in type II. According to the data from previous studies [2, 4, 8-10], small perforations can self-repair. These studies suggest treatment based the size and position of the perforation. Many

Table 2 Timing of implant placement by residual bone height

\begin{tabular}{lll}
\hline $\begin{array}{l}\text { Residual bone } \\
\text { height }(\mathrm{mm})\end{array}$ & $\begin{array}{l}\text { Simultaneous } \\
\text { (no. of implants) }\end{array}$ & $\begin{array}{l}\text { Delay (no. } \\
\text { of implants) }\end{array}$ \\
\hline$>7$ & 3 & 0 \\
$7-3$ & 40 & 0 \\
$<3$ & 49 & 7 \\
Total & 92 & 7 \\
\hline
\end{tabular}

Table 3 Types of implant surface

\begin{tabular}{lllc}
\hline Surface types & $\begin{array}{l}\text { Simultaneous placement } \\
\text { (no. of implants) }\end{array}$ & $\begin{array}{l}\text { Delayed placement } \\
\text { (no. of implants) }\end{array}$ & Total (\%) \\
\hline RBM & 51 & 6 & $57(57.6)$ \\
S\&E & 3 & 0 & $3(3)$ \\
SA & 38 & 1 & $39(39.4)$ \\
Total & 92 & 7 & $99(100)$ \\
\hline $\begin{array}{l}\text { RBM resorbable blasting media, S\&E sandblast and acid etched, SA sand } \\
\text { blasted with alumina and acid-etching }\end{array}$
\end{tabular}

studies $[6,10,14]$ suggest that perforation of the sinus can be treated with a resorbable membrane. Tiziano et al. [15] suggested that bioabsorbable membranes can be used to repair large perforations; in addition, they emphasized stabilization of the collagen membrane to repair perforated sinus membrane. In our study, we achieved successful surgical outcome after bone graft using resorbable collagen membrane and fibrin adhesive.

Bravetti et al. [16] reported that in elevation of the sinus membrane and insertion of bone graft or any other graft material, the Schneiderian membrane might be disrupted, and the graft material can be a source of chronic infection and sinusitis. On the other hand, Jensen et al. [5] reported that postoperative complications including infection and oro-antral fistula increased when a xenograft was used as the graft material. In this study, various materials used as a graft showed no impact on implant survival.

Some studies $[17,18]$ suggested that the implant with higher residual bone height $(>5 \mathrm{~mm}$ ) could have better primary stability and applicability for sinus elevation with simultaneous implant placement. Delayed implant placement was recommended for residual bone height of lower than $5 \mathrm{~mm}$. In this study, in cases of simultaneous implant placement, the residual bone height was an average of $3.4 \pm 2.0 \mathrm{~mm}$. In cases of delayed implant placement group, residual bone height was $0.6 \pm 0.9 \mathrm{~mm}$ on average. It may imply that primary stability is more important than residual bone height for determining the timing of implant placement.

Previous studies have reported acute maxillary sinusitis after sinus elevation of up to $26 \%[1,19,20]$. Some studies recommend prophylactic antibiotics and postoperative drug therapy to reduce infections [21, 22]. Another study [23] recommended the following regimens for complications of sinus elevation according to the patient group: for patients without allergy to penicillin, a combination of amoxicillin/clavulanic acid $1 \mathrm{~g}$ thrice a day (TID) and metronidazole $500 \mathrm{mg}$ TID per os for 7 to 10 days; and for patients allergic to penicillin, a regimen composed of levofloxacin $400 \mathrm{mg}$ BID per os until $72 \mathrm{~h}$ to symptom remission. In our study, development of a postoperative infection or maxillary sinusitis 
(14 cases) manifested as nasal congestion, headache, pain, fever, redness, or pain that worsened with bending forward with or without purulent drainage $[19,24,25]$ was treated with amoxicillin/clavulanic acid as the first-line drug. When there was no improvement of symptoms after taking amoxicillin/clavulanic acid for a week, metronidazole was added to the regimen as recommended, together with amoxicillin for an additional week. Radiographic examinations of these patients revealed maxillary sinus mucosal thickening, air-fluid levels, and radiographic signs, such as radiopacity. Additional medication was administered for approximately a week (range, 5 days to 4 weeks). Patients were informed about guidelines for basic maxillary postoperative care in addition to the antibiotics prescription. Mouth gargle with $0.1 \%$ chlorhexidine solution was also recommended. Ecchymosis along with mild bleeding (16 cases) and wound disruption (6 cases) were observed, but these symptoms showed no impact on the survival rate of implant.

An association between sinus perforation and graft dislodgement into the sinus with disruption of the normal sinus physiology has been previously described $[1,4,19,24$, 26, 27]. A disrupted mucociliary apparatus function and loss of the biologic barrier, caused by perforation of the membrane, can increase the invasion of bacteria into the sinus and cause infection [14, 28]. This may explain the increased incidence of secondary infections in our study.

The use of decongestants that may widen the ostia and improve nasal ventilation has been recommended [23]. However, chronic use of decongestants beyond 3 to 5 days should be discouraged, as they may result in significant rebound hyperemia and rhinitis medicamentosa. Horak et al. [29] reported that cetirizine (second-generation antihistamine)/pseudoephedrine is effective in the management of nasal congestion. In this study, we prescribed pseudoephedrine for cases with nasal congestion or rhinorrhea.

Successful implant placement is dependent on the achievement and maintenance of osseointegration [30]. Early studies with implant treatment relied on the placement of implants followed by healing period of 3 to 6 months during which the implants were protected from externally applied forces [31-34]. With the development of new implant types, surface technology, and advanced knowledge about the physiology of osseointegration, the requirement for delayed restoration of dental implants has been challenged [35-38]. However, implants placed in augmented sinus are different from conventional implants. Therefore, the timing of the implant loading is important for survival of implant placed in augmented sinus. Lang et al. [39] suggested that timing of sinus elevation and implant placement in relation to implant survival is affected by time of implant loading. Their results showed that implants that were immediately loaded regardless of the timing of the sinus elevation showed greater failure rates than implants in augmented bone that received a delayed loading protocol or those that were loaded immediately in sites that did not require a bone augmentation procedure. In this study, we applied the load on implant at least 5 months after implant placement and there was no failure.

Failure of bone graft or implant, which is commonly found in patients with perforated maxillary sinuses, was considered for additional debridement and irrigation or as the failure of the implant itself within one year of loading [40]. The data on implant survival rates vary by author. Proussaefs et al. [14] reported that implant success rate was $69.5 \%$ in the perforated maxillary sinus and $100 \%$ in the intact one, respectively. In the study by Khoury et al. [12], implant survival rate was lower when the membrane was perforated. On the other hand, some authors $[8,41]$ reported that maxillary sinus perforation does not have a negative effect on the success rate of implants. Schwartz-Arad et al. [7] also suggested that a maxillary sinus perforation affects postoperative complications such as sinusitis, but not the success rate of the implant. In this retrospective study, the survival rate of the implants in the patients who received sinus elevation of the perforated sinus membrane and implant placement with bone graft was $100 \%$.

The limitation of this study was that we included the implants that were not loaded. Potential limitations can arise from smoking or nonsmoking, differentiation of the sinus septa, or the systemic disease of the patients. Thus, for future studies, evaluation of the prognosis after loading and clinical outcomes by prognosis factor is necessary.

The results of our study suggested that the repair of a perforated sinus membrane using absorbable collagen membrane can be a safe and predictable procedure.

\section{Conclusions}

Sinus elevation can be successfully performed and produce a good outcome even in cases of abruptly perforated sinus membrane, regardless of the location or the size of the perforation. Repair using resorbable collagen membrane has predictable results and is a reliable technique. Further studies with a follow-up period for a long-term survival rate of implants in the maxillary sinus with perforated sinus membrane and confirmation of the present results are needed.

\section{Funding \\ None.}

Authors' contributions

KGS obtained the measurements and data, and wrote the manuscript. JWL helped in obtaining the data. JHC helped in drafting the manuscript. $J J H$ and SGJ have made substantial contributions to the analysis and interpretation of data. MSK, HJP, and SYR were involved in revising the manuscript. HKO participated in the study design and coordination and carefully reviewed and revised the manuscript. All authors read and approved the final manuscript. 


\section{Competing interests}

The authors declare that they have no competing interests.

\section{Ethics approval and consent to participate} None.

\section{Received: 20 October 2016 Accepted: 18 November 2016}

Published online: 05 December 2016

\section{References}

1. Misch CE (1987) Maxillary sinus augmentation for endosteal implants: organized alternative treatment plans. Int J Oral Implantol 4(2):49-58

2. Pikos MA (1999) Maxillary sinus membrane repair: report of a technique for large perforations. Implant Dent 8(1):29-34

3. Kasabah S, Krug J, Simunek A, Lecaro MC (2003) Can we predict maxillary sinus mucosa perforation? Acta Med (Hradec Kralove) 46(1):19-23

4. Van den Bergh JP, Ten Bruggenkate CM, Disch FJ, Tuinzing DB (2000) Anatomical aspects of sinus floor elevations. Clin Oral Implants Res 11(3):256-265

5. Jensen J, Sindet-Pedersen S, Oliver AJ (1994) Varying treatment strategies for reconstruction of maxillary atrophy with implants: Results in 98 patients. J Oral Maxillofac Surg 52:210-216

6. Vlassis JM, Fugazzotto PA (1999) A classification system for sinus membrane perforations during augmentation procedures with options for repair. J Periodontol 70(6):692-699

7. Schwartz-Arad D, Herzberg R, Dolev E (2004) The prevalence of surgical complications of the sinus graft procedure and their impact on implant survival. J Periodontol 75(4):511-516

8. Ardekian L, Oved-Peleg E, Mactei EE, Peled M (2006) The clinical significance of sinus membrane perforation during augmentation of the maxillary sinus. J Oral Maxillofac Surg 64(2):277-282

9. Betts NJ, Miloro M (1994) Modification of the sinus lift procedure for septa in the maxillary antrum. J Oral Maxillofac Surg 52(3):332-333

10. Fugazzotto PA, Vlassis J (2003) A simplified classification and repair system for sinus membrane perforations. J Periodontol 74(10):1534-1541

11. Cordioli G, Mazzocco C, Schepers E, Brugnolo E, Majzoub Z (2001) Maxillary sinus floor augmentation using bioactive glass granules and autogenous bone with simultaneous implant placement. Clinical and histological findings. Clin Oral Implants Res 12(3):270-278

12. Khoury F (1999) Augmentation of the sinus floor with mandibular bone block and simultaneous implantation: a 6-year clinical investigation. Int J Oral Maxillofac Implants 14(4):557-564

13. Hernández-Alfaro F, Torradeflot MM, Marti C (2008) Prevalence and management of Schneiderian membrane perforations during sinus-lift procedures. Clin Oral Implants Res 19(1):91-98

14. Proussaefs P, Lozada J, Kim J, Rohrer MD (2004) Repair of the perforated sinus membrane with a resorbable collagen membrane: a human study. Int J Oral Maxillofac Implants 19(3):413-420

15. Testori T, Wallace SS, Del Fabbro M, Taschieri S, Trisi P, Capelli M, Weinstein RL (2008) Repair of large sinus membrane perforations using stabilized collagen barrier membranes: surgical techniques with histologic and radiographic evidence of success. Int J Periodontics Restorative Dent 28(1):9-17

16. Bravetti P, Membre H, Marchal L, Jankowski R (1998) Histologic changes in the sinus membrane after maxillary sinus augmentation in goats. J Oral Maxillofac Surg 56(10):1170-1176

17. McCarthy C, Patel RR, Wragg PF, Brook IM (2003) Sinus augmentation bone grafts for the provision of dental implants: report of clinical outcome. Int J Oral Maxillofac Implants 18(3):377-382

18. Fugazzotto PA, Vlassis J (1998) Long-term success of sinus augmentation using various surgical approaches and grafting materials. Int J Oral Maxillofac Implants 13(1):52-58

19. Timmenga NM, Raghoebar GM, Boering G, van Weissenbruch R (1997) Maxillary sinus function after sinus lifts for the insertion of dental implants. J Oral Maxillofac Surg 55(9):936-939

20. Tidwell JK, Blijdorp PA, Stoelinga PJ, Brouns JB, Hinderks F (1992) Composite grafting of the maxillary sinus for placement of endosteal implants. A preliminary report of 48 patients. Int J Oral Maxillofac Surg 21(4):204-209

21. Esposito M, Cannizarro G, Bozzoli P, Checchi L, Ferri V, Landriani $S$ et a (2010) Effectiveness of prophylactic antibiotics at placement of dental implants: a pragmatic multicentre placebo-controlled randomised clinical trial. Eur J Oral Implantol 3(2):135-143
22. Esposito M, Grusovin MG, Loli V, Coulthard P, Worthington HV (2010) Does antibiotic prophylaxis at implant placement decrease early implant failures? A Cochrane systematic review. Eur J Oral Implantol 3(2):101-110

23. Testori T, Drago L, Wallace SS, Capelli M, Galli F, Zuffetti F et al (2012) Prevention and treatment of postoperative infections after sinus elevation surgery: clinical consensus and recommendations. Int J Dent 2012:365809

24. Manor Y, Mardinger O, Bietlitum I, Nashef A, Nissan J, Chaushu G (2008) Late signs and symptoms of maxillary sinusitis after sinus augmentation. Oral Surg Oral Med Oral Pathol Oral Radiol Endod 110(1):e1-e4

25. Sandler NA, Johns FR, Braun TW (1996) Advances in the management of acute and chronic sinusitis. J Oral Maxillofac Surg 54(8):1005-1013

26. Timmenga NM, Raghoebar GM, van Weissenbruch R, Vissink A (2003) Maxillary sinus floor elevation surgery: a clinical, radiographic and endoscopic evaluation. Clin Oral Implants Res 14(3):322-328

27. Pikos MA (2008) Maxillary sinus membrane repair: update on technique for large and complete perforations. Implant Dent 17(1):24-31

28. Zijderveld SA, van den Bergh JP, Schulten EA, ten Bruggenkate CM (2008) Anatomical and surgical findings and complications in 100 consecutivemaxillary sinus floor elevation procedures. J Oral Maxillofac Surg 66(7):1426-1438

29. Horak F, Toth J, Marks B, Stubner UP, Berger UE, Jager S et al (1998) Efficacy and safety relative to placebo of an oral formulation of cetirizine and sustained-release pseudoephedrine in the management of nasal congestion. Allergy 53(9):849-856

30. Branemark PI (1983) Osseointegration and its experimental backgraound. J Prosthet Dent 50(3):399-410

31. Adell R, Lekholm U, Rockler B, Branemark PI, Lindhe J, Eriksson B, Sbordone $L$ (1986) Marginal tissue reactions at osseointegrated titanium fixtures (I). A 3-year longitudinal prospective study. Int J Oral Maxilofac Surg 15(1):39-52

32. Barber HD, Seckinger RJ, Silverstein K, Abughazaleh K (1996) Comparison of soft tissue healing and ossointegration of IMZ implants placed in one-stage and two-stage techniques: A pilot study. Implant Dent 5(1):11-14

33. Bohsali K, Simon H, Kan JY, Redd M (1999) Modular transitional implants to support the interim maxillary overdenture. Compend Contin Educ Dent 20(10):975-978, 980, 982-983; quiz 984

34. Branemark PI, Hansson BO, Adell R, Breine U, Lindstrom J, Hallen O, Ohman A (1977) Osseointegrated implants in the treatment of the edentulous jaw. Experience from a 10-year period. Scand J Plast Reconstr Surg Suppl 16:1-132

35. Kan JY, Rungcharassaeng K, Lozada JL, Zimmerman G (2011) Facial gingival tissue stability following immediate placement and provisionalization of maxillary anterior single implants: A 2-to 8-year follow-up. Int J Oral Maxillofac Implants 26(1):179-187

36. Calvo MP, Muller E, Garg AK (2000) Immediate loading of titanium hexed screw-type implants in the edentulous patient: case report. Implant Dent 9(4):351-357

37. Raes F, Cosyn J, Crommelinck E, Coessens P, De Bruyn H (2011) Immediate and conventional single implant treatment in the anterior maxilla: 1-year results of a case series on hard and soft tissue response and aesthetics. J Clin Periodontol 38(4):385-394

38. Szmukler-Moncler S, Piattelli A, Favero GA, Dubruille JH (2000) Considerations preliminary to the application of early and immediate loading protocols in dental implantology. Clin Oral Implants Res 11(1):12-25

39. Lang LA, Edgin WA, Garcia LT, Olvera N, Verrett R, Bohnenkamp D, Hansey SJ (2015) Comparison of implant and provisional placement protocols in sinus-augmented bone: a preliminary report. Int J Oral Maxillofac Implants 30(3):648-656

40. Nolan PJ, Freeman K, Kraut RA (2014) Correlation between schneiderian membrane perforation and sinus lift graft outcome: a retrospective evaluation of 359 augmented sinus. J Oral Maxillofac Surg 72(1):47-52

41. Karabuda C, Arisan V, Ozyuvaci H (2006) Effects of sinus membrane perforations on the success of dental implants placed in the augmented sinus. J Periodontol 77(12):1991-1997 